
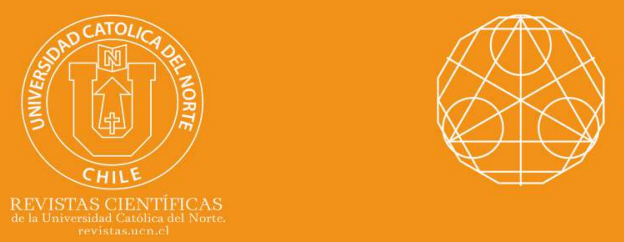

\title{
Implicative filters in quasi-ordered residuated systems
}

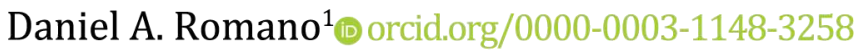

${ }^{1}$ International Mathematical Virtual Institute, Banja Luka, Bosnia and Herzegovina.

bato49@hotmail.com

\section{Abstract:}

The concept of residuated relational systems ordered under a quasiorder relation was introduced in 2018 by $S$. Bonzio and I. Chajda as a structure $\mathcal{A}=$ $\langle\mathrm{A}, \cdot, \rightarrow, 1, \mathrm{R}\rangle$, where $(A, \cdot)$ is a commutative monoid with the identity 1 as the top element in this ordered monoid under a quasi-order $R$. The author introduced and analyzed the concepts of filters in this type of algebraic structures. In this article, as a continuation of previous author's research, the author introduced and analyzed the concept of implicative filters in quasi-ordered residuated systems.

Keywords: Quasi-ordered residuated system; Implicative filter in quasiordered residuated system.

MSC (2020): 08A02, 06A11, 06B75.

Cite this article as (IEEE citation style):

D. A. Romano, "Implicative filters in quasi-ordered residuated systems", Proyecciones (Antofagasta, On line), vol. 40, no. 2, pp. 417-424, 2021, doi: 10.22199/issn.0717-6279-2021-020025

Article copyright: (C) 2021 Daniel A. Romano. This is an open access article distributed under the terms of the Creative Commons License, which permits unrestricted use and distribution provided the original author and source are credited. 


\section{Introduction}

Let $(A, \cdot, 1)$ be a commutative semigroup with the identity 1 . Suppose that on the carrier $A$ there exists another operation $\rightarrow$ and one relation $R$ that with multiplication in $A$ have a link $(x \cdot y, z) \in R \Longleftrightarrow(x, y \rightarrow z) \in R$ for each $x, y, z \in A$. A relational system designed in this way, when $R$ is a quasi-ordered relation on $A$, is in the focus of this paper.

The concept of residuated relational systems ordered under a quasiorder relation was introduced in 2018 by S. Bonzio and I. Chajda [2] in 2018. Previously, this concept was discussed in [1]. R. D. Maddux suggests that text [6] written by A. Tarski in 1941 is probably one of the first articles which relates to 'The calculus of relations' ([4], page 438). The approach outlined in [6] is worked out in more detail in [7]. According to R. D. Madduox already mentioned, the first definition of relation algebras appears in [3] (cited by [4], page 441). The approach outlined in [6] is worked out in more detail in [7]. In addition, according to R. D. Madduox, the first definition of relation algebras appears in [3] (cited by [4], page 441).

This paper continues the investigations of quasi-ordered residuated systems and of their filters which were started in the author article [5]. In particular, the concept of implicative filters in a quasi-ordered residuated system is introduced. Also, some conditions for a filter of such system to be an implicative filter are listed.

\section{Preliminaries}

\subsection{Concept of quasi-ordered residuated systems}

In article [2], S. Bonzio and I. Chajda introduced and analyzed the concept of 'residual relational systems'.

Definition 2.1. ([2], Definition 2.1) A residuated relational system is a structure $\mathcal{A}=\langle A, \cdot, \rightarrow, 1, R\rangle$, where $\langle A, \cdot, \rightarrow, 1\rangle$ is an algebra of type $\langle 2,2,0\rangle$ and $R$ is a binary relation on $A$ and satisfying the following properties:

(1) $(A, \cdot, 1)$ is a commutative monoid; (2) $(\forall x \in A)((x, 1) \in R)$; (3) $(\forall x, y, z \in A)((x \cdot y, z) \in R \Longleftrightarrow(x, y \rightarrow z) \in R)$.

We will refer to the operation - as multiplication, to $\rightarrow$ as its residuum and to condition (3) as residuation.

The basic properties for residuated relational systems are subsumed in the following 
Theorem 2.1 ([2], Proposition 2.1). Let $\mathcal{A}=\langle A, \cdot, \rightarrow, 1, R\rangle$ be a residuated relational system. Then

(4) $(\forall x, y \in A)(x \rightarrow y=1 \Longrightarrow(x, y) \in R)$,

(5) $(\forall x \in A)((x, 1 \rightarrow 1) \in R)$,

(6) $(\forall x \in A)((1, x \rightarrow 1) \in R)$,

(7) $(\forall x, y, z \in A)(x \rightarrow y=1 \Longrightarrow(z \cdot x, y) \in R)$,

(8) $(\forall x, y \in A)((x, y \rightarrow 1) \in R)$.

Recall that a quasi-order relation " on a set $A$ is a binary relation which is reflexive and transitive (Some authors use the term pre-order relation).

Definition 2.2. ([2], Definition 3.1) A quasi-ordered residuated system is a residuated relational system $\mathcal{A}=\langle A, \cdot, \rightarrow, 1$,$\rangle , where is a quasi-order$ relation in the monoid $(A, \cdot)$

The following proposition shows the basic properties of quasi-ordered residuated systems.

Proposition 2.1 ([2], Proposition 3.1). Let $A$ be a quasi-ordered residuated system. Then

(9) $(\forall x, y, z \in A)(x y \Longrightarrow(x \cdot z y \cdot z \wedge z \cdot x z \cdot y))$;

(10) $(\forall x, y, z \in A)(x y \Longrightarrow(y \rightarrow z x \rightarrow z \wedge z \rightarrow x z \rightarrow y))$;

(11) $(\forall x, y \in A)(x \cdot y x \wedge x \cdot y y)$.

Estimating that this topic is interesting $([1,2,5])$, it is certain that there is interest in the development of the concept of some substructures and processes in these systems.

Let $L(a)=\{y \in A: a y\}$ be the left class and $R(b)=\{x \in A: x b\}$ be the right class of the relation generated by the elements $a$ and $b$ respectively. Then $R(1)=A$. Some authors use the notation $U(a)$ instead of $L(a)$ (see, for example [2]).

\subsection{Concepts of filters}

In the article [5], in order to determine the concept of filters in quasi-ordered residuated systems, the relationships between the following conditions are analyzed: (F0) $1 \in F$; (F1) $(\forall u, v \in A)((u \cdot v \in F \Longrightarrow(u \in F \wedge v \in F))$; (F2) $(\forall u, v \in A)((u \in F \wedge u v) \Longrightarrow v \in F)$; and (F3) $(\forall u, v \in A)((u \in$ $F \wedge u \rightarrow v \in F) \Longrightarrow v \in F)$.

It is shown ([5], Proposition 3.2) that $(F 2) \Longrightarrow(F 1)$. In addition, it is shown ([5], Proposition 3.4) that for every nonempty subset of $F$ of system $\mathcal{A}$ is valid $(F 2) \Longrightarrow(F 0)$. Additionally, it has been shown to be valid 
Proposition 2.2 ([5], Proposition 3.6). Let $F$ be a submonoid of the monoid $(A, \cdot)$ in a quasi-ordered residuated system $\mathcal{A}=\langle(A, \cdot, \rightarrow, 1$,$\rangle . Then$ $F(2) \Longrightarrow F(3)$.

Based on our previous analysis of the interrelationship between conditions (F1), F(2) and (F3) in a quasi-ordered residual system, we introduced the concept of filters by the following definition.

Definition 2.3. ([5], Definition 3.1) For a non-empty subset $F$ of a quasiordered residuated system $\mathcal{A}$ we say that it is a filter of $\mathcal{A}$ if it satisfies conditions (F2) and (F3).

\section{The concept of implicative filters}

In this section, we introduce the concept of implicative filters in quasiordered residuared systems and analyze it.

Definition 3.1. For a non-empty subset $F$ of a quasi-ordered residuated system $\mathcal{A}$ we say that the implicative filter in $\mathcal{A}$ if (F2) and the following condition (IF) $(\forall u, v, z \in A)((u \rightarrow(v \rightarrow z) \in F \wedge u \rightarrow v \in F) \Longrightarrow u \rightarrow$ $z \in F$ )

are valid.

It is immediately seen that $1 \in F$ and $F$ satisfies condition (F1) because $F$ satisfies the condition (F2) and $F$ is a non-empty subset.

Proposition 3.1. Let $F$ be an implicative filter of a quasi-ordered residuated system $\mathcal{A}$. Then the following holds (F4) $(\forall u, v \in A)(u \rightarrow(u \rightarrow v) \in$ $F \Longrightarrow u \rightarrow v \in F)$.

Proof. If we put $v=u$ in (IF), we immediately obtain the claim of this proposition, since for every $u \in A$ always $u \rightarrow u \in F$ holds for every non-empty set $F$ satisfying condition (F2). Indeed, $u \rightarrow u \in F$ follows from $u u$; whence $1(u \rightarrow u)$ by $1 \in F$ and (F2).

In what follows, we need the following lemma

Lemma 3.1. Let a subset $F$ of a quasi-ordered residuated system $\mathcal{A}$ satisfies the condition (F2). Then the following holds (12) $(\forall u \in A)(u \in F \Longleftrightarrow$ $1 \rightarrow u \in F)$. 
Proof. Since $(\forall x \in A)(1 \rightarrow x x)$ and $(\forall x \in A)(x 1 \rightarrow x)$, by Proposition 2.3 (d) in [2], the proof of this lemma follows from (F2).

Let us show that every implicative filter in $\mathcal{A}$ is a filter of $\mathcal{A}$.

Theorem 3.1. Every implicative filter in a quasi-ordered residuated system $\mathcal{A}$ is a filter of $\mathcal{A}$.

Proof. Let $F$ be an implicative filter in a quasi-ordered residuated system $\mathcal{A}$. To prove that $F$ is a filter of $\mathcal{A}$, it suffices to prove that $F$ satisfies condition (F3). Let $u, v \in A$ be arbitrary elements such that $u \rightarrow v \in F$ and $u \in F$. Then $1 \rightarrow(u \rightarrow v) \in F$ and $1 \rightarrow u \in F$ by (12). Thus $1 \rightarrow v \in F$ by (IF). Hence $v \in F$ by (12). So, the set $F$ is a filter of $\mathcal{A}$.

We intend to more accurately describe this class of filters in quasiordered residuated systems. In what follows, we need the following two lemmas

Lemma 3.2. Let $F$ be a subset of a quasi-ordered residuated system $\mathcal{A}$. Then the condition (F2) is equivalent to the condition (F5) $(\forall u, v, z \in$ $A)((u \cdot v \in F \wedge u v \rightarrow z) \Longrightarrow z \in F)$.

Proof. $\quad(F 2) \Longrightarrow(F 5)$. Let $u, v, z \in A$ such that $x \cdot v \in F$ and $u v \rightarrow z$. Then $u \cdot v \in F \wedge u \cdot v z$ by (3). Thus $z \in F$ by (F2). (F5) $\Longrightarrow(F 2)$. Conversely, let us assume that (F4) holds. Let $u, v \in A$ such that $u \in$ $F \wedge u v$. Then $u \cdot 1 \in F \wedge u 1 \rightarrow v$. Thus $v \in F$ according (F5). So, the formula (F2) is proven.

Lemma 3.3. Let $a \in A$ be an element of a quasi-ordered residuated system $\mathcal{A}$. Then $L(a)=\{y \in A: a y\}$ is a filter of $\mathcal{A}$ if and only if the following holds (13) $(\forall u, v \in A)((a u \wedge a u v) \Longrightarrow a v)$.

Proof. Each set $L(a)$ satisfies condition (F2) according to Proposition 3.1 (13) in [5]. Let $u, v \in A$ be such $u \in L(a)$ and $u \rightarrow v \in L(a)$. Then $a u$ and $a u \rightarrow v$. Thus $a u$ and $a u v$ by (3). Hence $a v$ by (13), i.e. $v \in L(a)$. We have shown that $L(a)$ satisfies condition (F3). Therefore, $L(a)$ is a filter of $\mathcal{A}$. Conversely, it is obvious that condition (F3) is transformed into condition (13) in the case $F=L(a)$.

Another important result in this report is the following theorem

Theorem 3.2. In a quasi-ordered residuated system $\mathcal{A}$, the set $L(1)$ is an implicative filter if and only if $L(a)$ is a filter of $\mathcal{A}$ for any $a \in A$. 
Proof. Each set $L(a)$ satisfies condition (F2) according to Proposition 3.1 (13) in [5].

Assume that $L(a)$ is a filter of $\mathcal{A}$ for all $a \in A$. Let $u, v, z \in A$ be such that $u \rightarrow(v \rightarrow z) \in L(1)$ and $u \rightarrow v \in L(1)$. Then $1 u \rightarrow(v \rightarrow z)$ and $1 u \rightarrow v$. Thus $u \cdot v z$ and $u v$ by (3). Hence $u z$ by (13) because $L(u)$ is a filter of $\mathcal{A}$. So, $u \rightarrow z \in L(1)$. Therefore, the set $L(1)$ is an implicative filter.

Conversely, suppose that $L(1)$ is an implicative filter of $\mathcal{A}$. Let $u \in L(a)$ and $u \rightarrow v \in L(a)$. Then $a u \wedge a u \rightarrow v$ and $1 a \rightarrow u \wedge 1 a \rightarrow(u \rightarrow v)$. This means $a \rightarrow u \in L(1) \wedge a \rightarrow(u \rightarrow v) \in L(1)$. Since $L(1)$ is an implicative filter of $\mathcal{A}$, it follows $a \rightarrow v \in L(1)$. hence $1 a \rightarrow v$ and $a v$. So, $v \in L(a)$. Hence, $L(a)$ is a filter of $\mathcal{A}$.

The following theorem gives another condition for a filter of a quasiordered residuates system $\mathcal{A}$ to be an implicative filter in $\mathcal{A}$.

Theorem 3.3. Let $F$ be a filter of a quasi-ordered sesiduated system $\mathcal{A}$. Then $F$ is an implicative filter in $\mathcal{A}$ if and only if the set $F_{a}=\{x \in A$ : $a \rightarrow x \in F\}$ is a filter of $\mathcal{A}$ for any $a \in A$.

Proof. $\quad$ Note that $F_{1}=F$ by Lemma 3.1. (1) Assume that $F$ is an implicative filter in $\mathcal{A}$. Since $a 1$ by (2) for every $a \in A$, we have $1 a \rightarrow 1$ by (3). Then $1 \in F$ and $1 a \rightarrow 1$ implies $a \rightarrow 1 \in F$ by (F2). Thus $1 \in F_{a}$. Let us prove that $F_{a}$ satisfies condition (F2). Let $u, v \in A$ be such that $u \in F_{a}$ and $u v$. Then $a \rightarrow u \in F$ and $a \rightarrow u a \rightarrow v$ according to (10). Thus $a \rightarrow v \in F$ by (F2). Hence $v \in F_{a}$. Let $u, v \in A$ be such $u \in F_{a}$ and $u \rightarrow v \in F_{a}$. Then from $a \rightarrow u \in F$ and $a \rightarrow(u \rightarrow v) \in F$ it follows $a \rightarrow v \in F$ by (IF). Hence $v \in F_{a}$. This shows that $F_{a}$ satisfies condition (F3). So, since $F_{a}$ satisfies conditions (F2) and (F3), it is a filter of $\mathcal{A}$. (2) Conversely, suppose that $F_{a}$ is a filter of $\mathcal{A}$ for any $a \in A$. Let $u, v, z \in A$ be elements such that $u \rightarrow(v \rightarrow z) \in F$ and $u \rightarrow v \in F$. Then $v \rightarrow z \in F_{u}$ and $v \in F_{u}$. Thus $z \in F_{u}$ because $F_{u}$ is a filter of $\mathcal{A}$. Hence $u \rightarrow z \in F$. So, $F$ is an implicative filter in $\mathcal{A}$.

To design another condition equivalent to a condition (IF), we need the following lemmas

Lemma 3.4. Let a subset $F$ of a quasi-ordered residuated system $\mathcal{A}$ satisfy the condition (F2). Then the following holds (14) $(\forall u, v, z \in A)(u \rightarrow(v \rightarrow$ $z) \in F \Longleftrightarrow v \rightarrow(u \rightarrow z) \in F)$. 
Proof. Let $u, v, z \in A$ be elements such that $u \rightarrow(v \rightarrow z) \in F$. From here and from the condition $(\forall u, v, z \in A)(u \rightarrow(v \rightarrow z) v \rightarrow(u \rightarrow z)$ ([2], Proposition 3.1(f)) follows $v \rightarrow(u \rightarrow z) \in F$. according to (F2). Reverse implication is obtained from previously proven if variables $u$ and $v$ change places.

Lemma 3.5. Let $A$ be a quasi-ordered residuated system. Then (15) $(\forall x, y, z \in A)(y \rightarrow z(x \rightarrow y) \rightarrow(x \rightarrow z))$.

Proof. In [2] ((Proposition 3.1(g)) is shown that the following

$$
(\forall x, y, z \in A)((x \rightarrow y) \cdot(y \rightarrow z)(x \rightarrow z))
$$

holds. Since multiplication in $\mathcal{A}$ is commutative, we have

$$
(\forall x, y, z \in A)((y \rightarrow z) \cdot(x \rightarrow y)(x \rightarrow z)) .
$$

From here we get (15) according to (3).

Theorem 3.4. Let $F$ be a non-empty subset of a quasi-ordered residuated system $\mathcal{A}$ satisfying (F2). If $F$ satisfies the additional condition (F6) $(\forall u, v, z \in A)((u \rightarrow(v \rightarrow(v \rightarrow z)) \in F \wedge u \in F) \Longrightarrow v \rightarrow z \in F)$

then $F$ is an implicative filter in $\mathcal{A}$.

Proof. Let us show that the set $F$ satisfies condition (FI). Let $u, v, z \in A$ be arbitrary elements such that $u \rightarrow(v \rightarrow z) \in F$ and $u \rightarrow v \in F$. Using (14) and (15) we have

$$
v \rightarrow(u \rightarrow z) \in F \text { and } v \rightarrow(u \rightarrow z)(u \rightarrow v) \rightarrow(u \rightarrow(u \rightarrow z)) .
$$

Thus

$$
(u \rightarrow v) \rightarrow(u \rightarrow(u \rightarrow z)) \in F \wedge u \rightarrow v \in F .
$$

Hence $u \rightarrow z \in F$ by (F6). So, $F$ is an implicative filter.

We conclude this report with the following theorem

Theorem 3.5. If a non-empty subset $F$ of a quasi-ordered residuates system $\mathcal{A}$ satisfies conditions (F2), (F3) and (F4), then $F$ is an implicative filter in $\mathcal{A}$.

Proof. To prove that $F$ is an implicative filter in $\mathcal{A}$, it suffices to show that (F6) is a valid formula. Let $u, v, z \in A$ be such that $u \rightarrow(v \rightarrow(v \rightarrow$ $z)) \in F$ and $u \in F$. Then $v \rightarrow(v \rightarrow z) \in F$ and $u \in F$ by (F3). Thus $v \rightarrow z \in F$ by (F4). 


\section{Competing Interests.}

The author declares that there is none conflict of interest regarding the publication of this manuscript.

\section{Acknowledgment}

The author thanks the reviewer for helpful suggestions that ideas presented in the text getting a consistent form.

\section{References}

[1] E. Bonzio, "Algebraic structures from quantum and fuzzy logics", $\mathrm{PhD}$. Thesis, Università degli studi di Cagliari, 2016. [On line]. Available: https:/ / bit.ly/ 3bUmb5N

[2] S. Bonzio and I. Chajda, "Residuated relational systems", Asian-European journal of mathematics, vol. 11, no. 2, Art ID. 1850024, 2018, doi: 10.1142/ S1793557118500249

[3] B. Jónsson and A. Tarski, "Representation problems for relation algebras" [Abstract], in Bulletin of the American Mathematical Society, 1948, vol. 54, no. 1, p. 80, doi: 10.1090/ S0002-9904-1948-08948-0

[4] R. D. Maddux, "The origin of relation algebras in the development and axiomatization of the calculus of relations", Studia logica, vol. 50, no. 3, pp. 421-455, 1991, doi: 10.1007/ BF00370681

[5] D. A. Romano, "Filters in residuated relational system ordered under quasi-order", Bulletin International Mathematics Virtual Institute, vol. 10, no. 3, pp. 529-534, 2020, doi: 10.7251/ BIMVI2003529R

[6] A. Tarski, "On the calculus of relations", The journal of symbolic logic, vol. 6, no. 3, pp. 73-89, 1941, doi: 10.2307/ 2268577

[7] A. Tarski and S. R. Givant, A formalization of set theory without variables. Providence, RI: AMS, 1987. 